\title{
Uso sustentável da biodiversidade e conservação de recursos naturais
}

\author{
Sustainable use of biodiversity and conservation of natural resources
}

\author{
Luiz Everson da Silva ${ }^{1}$ \\ Ulysses Paulino de Albuquerque ${ }^{2}$ \\ Wanderlei do Amaral ${ }^{3}$
}

\section{Introdução}

Neste número da GUAJU - Revista Brasileira de Desenvolvimento Territorial Sustentável trazemos à reflexão de estudantes, pesquisadores e comunidade, os aspectos conjunturais e analíticos que traduzam as inquietudes sobre o uso sustentável da biodiversidade e a conservação dos recursos naturais em articulação com o Desenvolvimento Territorial. A ideia deste número temático foi possibilitar a reflexão sobre o sistema de ciência e tecnologia regional, primando por artigos e pesquisas focalizados no desenho e no teste de modelos de territórios sustentáveis para os diferentes tipos de regiões no país. Recebemos artigos relacionados a estudos de caso, exemplos de processo de agregação de produtos da biodiversidade com o intuito de geração de trabalho e renda para as comunidades locais, além de relatos sobre a cooperação possível entre os saberes tradicionais e a ciência. Tudo isso com o intuito de comunicar uma conservação da biodiversidade inspirada por valores para além do mercado.

O presente número da revista é fruto da discussão iniciada no Fórum de Pesquisa sobre o Uso Sustentável da Biodiversidade e Conservação de Recursos Naturais realizado em novembro de 2016 em Matinhos - Região Litorânea do Paraná. Na oportunidade estavam reunidos pesquisadores, representantes do Instituto Chico Mendes de Conservação da Biodiversidade - ICMBIO e estudantes que juntos refletiram sobre crescimento sustentável do Estado, especificamente do litoral paranaense. Apontou-se a necessidade do estudo das riquezas e desta biodiversidade, sem esquecer o crescimento agrícola e o agronegócio e a sustentabilidade local. Ficou claro o papel da aliança dos diferentes atores

\footnotetext{
${ }^{1}$ Doutor em Química. Professor do Programa de Pós-Graduação em Desenvolvimento Territorial Sustentável da UFPR. E-mail:luiz_everson@yahoo.de

${ }^{2}$ Doutor em Biologia Vegetal.Professor Associado do Departamento de Botânica da UFPE - Pesquisador 1A do CNPq. E-mail:upa677@hotmail.com

${ }^{3}$ Doutor em Agronomia.Servidor Técnico do Departamento de Engenharia Química da UFPR - Bolsista PNPD do PPGDTS 2015/2017. E-mail:wdoamaral@ hotmail.com
} 
no intuito de fortalecer os estudos e conduzir ações em conjunto, evitando com isso a mercantilização dos recursos naturais.

\section{A ciência tem tentado compreender as relações socioecológicas no contexto do uso da biodiversidade}

O conceito contemporâneo de diversidade biológica procura referir e integrar toda a variedade e variabilidade que encontramos em organismos vivos, nos seus diferentes níveis, e os ambientes nos quais estão inseridos. A Convenção sobre Diversidade Biológica (CDB) incorporou algumas destas novas tendências na sua conceituação de diversidade biológica, a qual é definida em seu art. $2^{\circ}$, in verbis, como:

a variabilidade de organismos vivos de todas as origens, compreendendo, dentre outros, os ecossistemas terrestres, marinhos e outros ecossistemas aquáticos e os complexos ecológicos de que fazem parte, compreendendo ainda a diversidade dentro de espécies, entre espécies e de ecossistemas (BRASIL,2000).

Por outro lado, é sabido que atingimos um limite quanto aos recursos disponíveis em nível local e global e que a questão do modelo de desenvolvimento para as próximas gerações depende, entre outras coisas, dos serviços proporcionados pelo sistema natural (clima, solo, ecologia etc.). Logo, a influência antrópica nos processos globais de clima, circulação de nutrientes, deslocamento de espécies, entre outras, tem mobilizado cientistas ao redor do mundo no intuito de reconhecer e compreender que o uso que se faz dos ativos da biodiversidade está embutido no sistema socioeconômico e traz em seu bojo valores, relações sociais e políticas, direito de uso, leis, governança, mercado e relações econômicas. Por isso a importância de se estudar as relações socioecológicas integrando os componentes socioeconômicos e biofísicos.

Temos alguns exemplos de sistema socioecológico, entre eles podemos destacar o estudo conduzido na Floresta Nacional do Araripe - Sertão Cearense, onde múltiplos grupos de interesse (23 comunidades, entre elas agricultores, pecuaristas e extrativistas) interagem em busca de múltiplos objetivos (produção, lucro, equidade, conservação e manutenção cultural); onde os fatores biofísicos (como água, clima e biodiversidade) afetam e são afetados por essas atividades sociais e econômicas; e onde múltiplos fatores, internos e externos, vindo das escalas locais, nacionais e internacionais (desde políticas públicas e aplicação das leis, à cultura, poder e eficácia de diferentes discursos) influenciam sua dinâmica. 
Assim, foi possível, através da integração dos diferentes atores, mediados pela pesquisa de 16 estudantes de mestrado e doutorado, conduzir estudos sobre vários aspectos relacionados à história de ocupação desta área, uso da Floresta e manejo dos recursos naturais.

Metodologicamente falando, identificou-se a cadeia produtiva da sociobiodiversidade em seus diferentes aspectos:

a) seleção das comunidades envolvidas no extrativismo da Floresta Nacional;

b) análise das relações socioambientais no trabalho extrativista;

c) análise da realidade socioeconômica das comunidades extrativistas;

d) seleção das cadeias produtivas de maior importância local;

e) estudo efetivo das cadeias produtivas;

f) proposta de ação para a conservação dos recursos naturais.

Quando pesquisadores, tomadores de decisão e atores sociais tentam gerenciar sistemas socioecológicos, ou mesmo compreender o seu funcionamento, as perspectivas de economistas, advogados, cientistas sociais e ecólogos, entre outros, são válidas, mas depender de qualquer uma dessas perspectivas exclusivamente é insuficiente, pois os parâmetros e limitações de outras áreas irão sobrecarregar os esforços se não forem adequadamente considerados. Torna-se necessário, portanto, criar teorias, conceitos, ferramentas e metodologias para enxergar o sistema como um todo e entender seus processos de mudança.

\section{O desafio de conciliar conservação com uso e manejo dos recursos naturais}

É consenso que a conservação e a preservação da diversidade biológica garantem a sobrevivência do planeta como um todo. Permite-nos a manutenção e recuperação da vida. Portanto, torna-se uma necessidade estratégica dos países detentores de tecnologias mais avançadas, resguardarem esse patrimônio, visto que mudanças climáticas globais, movimentos de continentes, erupções vulcânicas, choques de meteoros, entre outros fatores, podem alterar drasticamente a vida sobre a Terra.

Por outro lado, os componentes da biodiversidade são passíveis de fornecer ampla gama de produtos de importância econômica. Neste sentido, o resgate do valor nutricional dos produtos tornase imperativo. Assim, políticas integradoras na esfera nacional e internacional têm sido conduzidas no intuito de garantir a proteção da biodiversidade aliado à agricultura como pontos fundamentais no combate à fome. 
Outra demanda está relacionada aos medicamentos disponíveis na terapêutica oriundos de fontes naturais, sejam elas plantas, micro-organismos e animais. Neste campo, pesquisas em diversos países têm sido conduzidas para garantir a incorporação de fitomedicamentos no mercado farmacêutico. Destaca-se ainda o mercado cosmético e o avanço da biotecnologia como usuários de produtos e serviços relacionados à biodiversidade.

Neste contexto, e em função da diversidade vegetal de cada região, ganha corpo pesquisas que contemplem a associação entre o recurso genético disponível na floresta, a diversidade cultural local e o uso sustentável dos recursos naturais. Nesta esfera, o conhecimento tradicional, oriundo de populações tradicionais, tem o papel de subsidiar as pesquisas que permitam o surgimento de novos medicamentos ou entidades químicas que possam ser utilizados como fitoterápicos, entre outros usos.Porém se faz necessária a formulação de estratégias de conservação, domesticação e desenvolvimento de pesquisas com espécies nativas, no sentido de garantir que a pressão sofrida pelo extrativismo seja substituída por uma gestão agrícola e manejo sustentável. Esta ação visa subsidiar produtores familiares no sentido de possibilitar o surgimento de trabalho e renda a partir dos produtos agroflorestais, mas numa perspectiva de desenvolvimento territorial sustentável.

Por outro lado, corroboramos com Albuquerque ao afirmar que as pesquisas realizadas a partir de recursos genéticos de espécies medicinais nativas, somente serão aplicadas se seu material genético estiver seguro quanto à sobrevivência e disponibilidade. Por isso são necessários estudos que investiguem a melhor forma de propagação para que se desenvolva seu cultivo e manejo (ALBUQUERQUE et al., 2002).

Sabemos que a investigação dos componentes e substâncias contidas nas plantas, sua caracterização e análise em laboratório, contém um grande potencial de agregar valor às práticas já realizadas nas comunidades rurais, indígenas e tradicionais, bem como a geração de trabalho e renda para essas comunidades. Além disso, tem como potencialidade também a popularização de fitoterápicos nas unidades de saúde.

Dentre as estratégias para o levantamento do conhecimento sobre plantas medicinais, a abordagem etnofarmacológica combina informações adquiridas junto às populações tradicionais que utilizam da flora local para o tratamento de doenças com estudos químicos e farmacológicos em laboratórios (ELISABETSKY; SOUZA, 2007).

Vale ressaltar que a Etnofarmacologia é uma disciplina de caráter multi e interdisciplinar, envolvendo profissionais de diversas áreas do conhecimento. De acordo com Elisabetsky e Souza (2007) um estudo etnofarmacológico envolve várias etapas:

1) Coleta e análise dos dados etnofarmacológicos; 
2) Identificação botânica do material, incluindo depósito de material-testemunha em herbário;

3) Pesquisa bibliográfica em bancos de dados de química e biologia em geral, e de plantas medicinais em particular, tais como Chemical Abstracts, Biological Abstracts, Index Medicus, IPA, NAPRALERT, entre muitos outros. Os dados quimiotaxonômicos podem sugerir a presença de uma (ou mais) determinada classe de substâncias químicas, a (s) qual (is) torna (m) -se objeto de hipóteses quanto à possível participação na atividade biológica;

4) Análise química preliminar para detectar as classes de compostos presentes na parte da planta usada medicinalmente e na própria preparação tradicional; a interpretação do significado químico do modo de preparo popular é útil na definição de marchas fitoquímicas para obtenção dos extratos iniciais, já que as substâncias ativas devem estar presentes na preparação que é administrada aos usuários

5) Estudo farmacológico preliminar do (s) estrato (s) bruto (s) em modelos experimentais relevantes relacionados à (s) ação (ões) farmacológica (s) sugerida (s) pela análise da (s) informação (ões) popular (es);

6) Fracionamento químico por métodos diversos (cromatográfico, diferenças de solubilidade, gradiente de $\mathrm{pH}$, cristalizações sucessivas, etc.), monitorado com base nos resultados das análises farmacológicas; as frações de interesse farmacológicas; as frações de interesse farmacológico são mais profundamente estudadas com uso de técnicas analíticas (CCD, CG, CLAE, reações específicas);

7) Estudo farmacológico abrangente e toxicologia pré-clínica de frações padronizadas, e/ou compostos isolados, e/ou formulação farmacêutica a ser comercializada, para subsidiar os estudos clínicos;

8) Elucidação das estruturas das substâncias ativas isoladas e/ou obtenção de derivados (UV, IV, RMN, ${ }^{1} \mathrm{H} \mathrm{e}{ }^{13} \mathrm{C}, \mathrm{EM}$ ) orientam a farmacotécnica e o controle de qualidade químico/biológico, além de fundamentar futuros estudos farmacológicos com substâncias ativas semissintéticas (ELIZABETSKI; SOUZA, 2007, p.109-110).

Independente das práticas de manejo ocorrerem em ambientes aquáticos ou terrestres, os elementos da biodiversidade manejados passam a ser reconhecidos não apenas por suas características naturais, mas pela relação direta com o humano que os maneja. A sociobiodiversidade carrega a marca da intervenção humana, contudo não é qualquer forma de interação entre homem e natureza, trata-se de uma relação estabelecida ao longo do tempo em que há saberes específicos e técnicas de manejo.

\section{Conflitos socioambientais e os desafios de gerir os recursos naturais}

As práticas, costumes, conhecimentos e valores de comunidades tradicionais estão imbricados na forma como se relacionam entre si e com a biodiversidade dos ecossistemas que estão inseridos, bem como a biodiversidade que manejam para sua subsistência. Diegues (2004, p.33) fala sobre a maneira como as populações tradicionais manejam e até domesticam a biodiversidade, e ao mesmo tempo atribuem ao conjunto de seres vivos um valor de uso e um valor simbólico. "As populações tradicionais não só convivem com a biodiversidade, mas nomeiam e classificam as espécies vivas segundo suas próprias categorias e nomes". 
Na medida em que estas comunidades fazem o uso da terra, da água, das florestas - geralmente de modo comum, sem a propriedade individual do recurso - constituem, assim, seus territórios tradicionais. Haesbaert (2004) afirma que o conceito de território não se restringe a dimensão material (terra), o conceito de território demanda uma concepção integradora que envolve tanto sua dimensão política (relações de poder), quanto econômica (usos), cultural (simbólico) e natural (terra, água, vegetação). O território configura-se como um espaço definido e delimitado por e a partir de relações de poder (SOUZA, 2003) fruto dos sujeitos, ou seja, são esses que produzem o território, partindo da realidade inicial dada, que é o espaço (RAFFESTIN, 1993).

Práticas como a agricultura itinerante e o extrativismo vegetal e animal, como ainda hoje são praticadas por populações tradicionais, configuram-se como sistemas tradicionais de manejo. Possuem um conjunto de saberes e técnicas que tem contribuído significativamente com a manutenção da diversidade biológica. Diegues e Arruda (2001) afirmam que a biodiversidade não é simplesmente um produto da natureza, mas em muitos casos é produto da ação das sociedades e culturas humanas, em particular, das sociedades tradicionais não industriais. Para os autores, as culturas e saberes tradicionais podem contribuir com a manutenção da biodiversidade dos ecossistemas, visto que, em numerosas situações, esses saberes se configuram como o resultado de uma co-evolução entre as sociedades e seus ambientes naturais.

Para identificar as espécies manejadas por comunidades tradicionais, bem como as formas de uso dos ecossistemas pelas comunidades tradicionais e principalmente para reconhecer os saberes tradicionais envolvidos nestas práticas, podemos adotar a Etnoecologia como suporte teórico e metodológico. Para Marques (2001, p. 37),

a Etnoecologia é o campo de pesquisa (científica) transdisciplinar que estuda os pensamentos (conhecimentos e crenças), sentimentos e comportamentos que intermediam as interações entre as populações humanas que os possuem e os demais elementos dos ecossistemas que as incluem, bem como os impactos ambientais daí decorrentes.

Assim, entendemos que discutir estratégias de Desenvolvimento Territorial Sustentável para as comunidades, passa por um olhar sobre os processos de reprodução social dos povos nativos, em consonâncias com os meios de vida da comunidade, principalmente na tentativa de entender as várias dimensões da relação com a biodiversidade. Garantindo também a dimensão territorial condizente com a territorialidade historicamente construída através das práticas tradicionais e novas práticas (políticas, técnicas) necessárias à garantia de direitos desses sujeitos. 


\section{Os desafios da conservação}

Por fim, o desafio de pensar a conservação de uma determinada região passa pela operacionalização de projetos elaborados para esta localidade com equidade social, sustentabilidade ambiental em todas as suas dimensões: social, cultural, ecológica, ambiental, territorial, econômica, políticas nacional e internacional (SACHS, 2008). Numa tentativa de encaminhar algumas ações, nos atrevemos a apontar algumas pistas para contribuir com o debate e suscitar novos encaminhamentos. O primeiro passa pela necessidade de gerar oportunidades de diálogo entre gestores de Unidades de Conservação (UC's) e usuários da biodiversidade.

Numa outra esfera, entendemos que continuar investigando em profundidade as diferentes relações entre seres humanos, recursos e território, também tem se mostrado uma estratégia viável. Tal ação pode, através da sinergia dos diferentes atores, produzir conhecimento científico e técnico relevante para tomada de decisões. Tudo isso para criar oportunidades no enfrentamento dos problemas.

A pergunta permanece: como cumprir com as necessidades humanas sem acabar com os serviços e processos essenciais da natureza? Alternativas existem, seja em nível individual, familiar ou em pequenos grupos, podemos destacar: diversificar fontes de renda, investir nas relações sociais, manter reservas de capital de diferentes tipos, desenvolver competências de aprendizagem e adaptação. Porém todas essas ainda estão assombradas pela escala sistêmica - um colapso econômico, político ou ambiental.

Acreditamos que no sistema complexo que vivemos, grande parte das soluções virá de escalas menores do sistema - atores individuais e iniciativas locais testadas e demonstradas. Mas o sistema precisará, também, de mecanismos para disseminar e replicar pequenos sucessos. Além disso, o contexto institucional e os processos que acontecem nas macroescalas do sistema vão criar, ou não, um contexto adequado para esta exploração e replicação local. Portanto, precisa-se buscar soluções em todos os níveis.

Diante desse quadro, relatamos abaixo as contribuições recebidas para esse Dossiê Temático. No primeiro artigo temos a contribuição de Débora Moreira de Oliveira, Daniela Silva Cruz, Bruno Antônio Lemos de Freitas, Thaiane Natalle Moreira Lima e Laura Jane Gomes, pesquisadores ligados ao Programa em Desenvolvimento e Meio Ambiente da Universidade Federal de Sergipe. O artigo intitulado Identificação dos pontos críticos no sistema extrativista da mangaba (Hancornia speciosa Gomes) em Sergipe relata os pontos críticos do sistema extrativista da Mangaba. Este estudo foi 
conduzido com o intuito de subsidiar a seleção de indicadores de sustentabilidade para o monitoramento da cadeia produtiva da espécie.

O segundo artigo intitulado A política de garantia de preços mínimos para produtos da sociobiodiversidade (PGPM-BIO): potencialidades da intervenção estatal para a preservação ambiental em Goiás nos traz a reflexão sobre a política de preços mínimos da sociobiodiversidade (PGPM-BIO) em Goiás. Os autores - Camila Vito Silva de Lima, Hamilton Matos Cardoso Júnior e Divina Aparecida Leonel Lunas - inferem sobre a dependência do mapeamento das áreas produtivas, da ampla divulgação da política e da formalização da cadeia produtiva, por meio da consolidação dos mercados para os produtos da sociobiodiversidade.

Pesquisadores do Tocantins em seu artigo Envolvimento Ambiental, caminho sustentável e a defesa da sociobiodiversidade na Reserva Extrativista do Extremo Norte do Tocantins relatam a experiência das quebradeiras de coco da reserva Extrativista do Norte Tocantins. Fábio Pessoa Vieira e Lucas Barbosa Souza destacam as experiências vividas e os diversos saberes associados ao território.

Uma abordagem interessante é feita no artigo Conflitos em áreas de preservação permanente na bacia hidrográfica do Rio Pitimbu- RN: proposta de um indicador institucional sustentável. Os autores Vinícius Meressiev Melo de Oliveira e Leci Martins Menezes Reis definem um indicador de sustentabilidade denominado taxa de conflito em APP, mediante a correlação entre as áreas de preservação permanente e de cobertura do solo. Pode-se constatar, através do cruzamento entre as áreas de APP e as classes de uso do solo, áreas de conflito e processos de degradação constante.

No artigo Desenvolvimento Rural no Cerrado, desenvolvimento e envolvimento das famílias agroextrativistas, Sued Wilma Caldas Melo analisa o desenvolvimento rural no bio Cerrado a partir das estratégias de produção e reprodução social. A questão do agroextrativismo é, segundo a autora, uma forma alternativa de uso dos recursos do Cerrado com potencialidades ambientais, sociais e econômicas.

Por último, o texto Uso de Ferramentas de Adaptação baseada em Ecossistemas para Mudanças Climáticas: revisão da literatura é apresentado. Os autores Alexandre Dullius e Maclovia Corrêa da Silva, buscaram em artigos, teses e dissertações o estado da arte sobre o tema. Constatouse que a ferramenta mais utilizada é a que trata da adaptação baseada em Ecossistema (Abe), pois utiliza recursos da natureza como forma de adaptação. 


\section{Referências}

ALBUQUERQUE, Ulysses Paulino de; ANDRADE, Laise de Holanda Cavalcanti. Conhecimento botânico tradicional e conservação em uma área de caatinga no estado de Pernambuco, Nordeste do Brasil. Acta Bot. Bras., vol.16, no.3, p.273-285, jul./set. 2002.

BRASIL, MINISTÉRIO DO MEIO AMBIENTE (MMA). Convenção sobre Diversidade Biológica - CDB. Brasília, 2000.

DIEGUES, Antônio Carlos e ARRUDA, Rinaldo Sérgio Vieira. Saberes tradicionais e biodiversidade no Brasil. Brasília: Ministério do Meio Ambiente, 2001.

DIEGUES, Antônio Carlos. Saberes Tradicionais e Etnoconservação. In: Comunidades tradicionais e manejo dos recursos naturais da Mata Atlântica. DIEGUES, A. C. e VIANA, V. M. (org). 2. ed. - São Paulo : HUCITEC: NUPAUB, 2004. p 9 - 22.

ELISABETSKY, E.; SOUZA, G. C. Etnofarmacologia como ferramenta na busca de substâncias ativas. In: SIMÕES, C. M. O. et al. (Org.). Farmacognosia: da planta ao medicamento. 6. ed. Porto Alegre: UFRGS: UFSC, 2007. p. 107-122.

HAESBAERT, Rogério. O mito da desterritorialização: do "Fim dos Territórios" à Multiterritorialidade. 2. ed. Rio de Janeiro: Bertrand Brasil, 2004.

MARQUES, José Geraldo Wanderley. O Olhar (Des)Multiplicado. O Papel do Interdisciplinar e do Qualitativo na Pesquisa Etnobiológica e Etnoecológica. In: Amorozo, M.C.M., Ming, L.C., Silva, S.M.P. (eds.) Métodos de Coleta e Análise de Dados em Etnobiologia, Etnoecologia e Disciplinas Correlatas. Seminário de Etnobiologia e Etnoecologia do Sudeste. Anais... Rio Claro/SP: Coordenadoria de Área de Ciências Biológicas. UNESP/CNPq. 2001. p. 31-46.

RAFFESTIN, Claude. Por uma geografia do poder. São Paulo: Ática, 1993.

SACHS, I. (2008). Desenvolvimento: Includente, Sustentável, Sustentado. Rio de Janeiro: Garamond (152 p.).

SOUZA, Marcelo José Lopes de. O território: sobre o espaço e poder, autonomia e desenvolvimento. In: CASTRO, Iná Elias de; GOMES, Paulo César da Costa; CÔRREA, Roberto Lobato (Org.). Geografia: conceitos e temas. 3. ed. Rio de Janeiro: Bertrand Brasil, 2001. 